Volume 32 (2022) 62-79

DOI: $10.24330 /$ ieja.1081701

\title{
GRADED S-1-ABSORBING PRIME SUBMODULES IN GRADED MULTIPLICATION MODULES
}

\author{
F. Farzalipour and P. Ghiasvand \\ Received: 25 February 2021; Revised: 2 August 2021; Accepted: 29 September 2021
}

Communicated by Meltem Altun Özarslan

\begin{abstract}
Let $G$ be a group with identity $e$. Let $R$ be a commutative $G$ graded ring with non-zero identity, $S \subseteq h(R)$ a multiplicatively closed subset of $R$ and $M$ a graded $R$-module. In this article, we introduce and study the concept of graded $S$-1-absorbing prime submodules. A graded submodule $N$ of $M$ with $\left(N:_{R} M\right) \cap S=\emptyset$ is said to be graded $S$-1-absorbing prime, if there exists an $s_{g} \in S$ such that whenever $a_{h} b_{h^{\prime}} m_{k} \in N$, then either $s_{g} a_{h} b_{h^{\prime}} \in\left(N:_{R} M\right)$ or $s_{g} m_{k} \in N$ for all non-unit elements $a_{h}, b_{h^{\prime}} \in h(R)$ and all $m_{k} \in h(M)$. Some examples, characterizations and properties of graded $S$-1-absorbing prime submodules are given. Moreover, we give some characterizations of graded $S$-1-absorbing prime submodules in graded multiplicative modules.
\end{abstract}

Mathematics Subject Classification (2020): 16W50, 13C05, 16D80

Keywords: Graded S-1-absorbing prime ideal, graded S-1-absorbing prime submodule, graded multiplication module

\section{Introduction}

The study of graded rings arises naturally out of the study of affine schemes and allows them to formalize and unify arguments by induction. However, this is not just an algebraic trick. The concept of grading in algebra, in particular graded modules is essential in the study of homological aspect of rings. Much of the modern development of the commutative algebra emphasizes graded rings. Graded rings play a central role in algebraic geometry and commutative algebra. Gradings appear in many circumstances, both in elementary and advanced level. In recent years, rings with a group-graded structure have become increasingly important and consequently, the graded analogues of different concepts are widely studied (see [1], [4], [7], [8], [9], [10], [11], [12], [13] and [14]). In the third section of this paper, we introduce and study the notion of graded $S$-1-absorbing prime submodules of a graded $R$-module $M$ as a generalization of graded prime submodules and we investigate some properties of such graded submodules. For example, we show that 
if $N$ is a graded $S$-1-absorbing prime submodule of $M$, then $\left(N:_{R} M\right)$ is a graded $S$-1-absorbing prime ideal of $R$, but the converse is not true. Also, we prove that $N$ is a graded $S$-1-absorbing prime submodule of $M$ if and only if there exists an $s_{g} \in S$ such that whenever $I J K \subseteq N$, then either $s_{g} I J \subseteq\left(N:_{R} M\right)$ or $s_{g} K \subseteq N$ for all graded ideals $I, J$ of $R$ and all graded submodules $K$ of $M$. In the last section, we give some characterizations of graded $S$-1-absorbing prime submodules in graded multiplicative modules. Most of the results in this article are inspired from [6].

Throughout this work, all graded rings are assumed to be commutative graded rings with identity, and all graded modules are unitary graded $R$-modules.

\section{Preliminaries}

In this section we state some definitions and results of graded rings and graded modules which we need to develop our paper.

Definition 2.1. [14] Let $G$ be a group with identity $e$ and $R$ be a ring. Then $R$ is said to be $G$-graded if $R=\bigoplus_{g \in G} R_{g}$ such that $R_{g} R_{h} \subseteq R_{g h}$ for all $g, h \in G$, where $R_{g}$ is an additive subgroup of $R$ for all $g \in G$.

The elements of $R_{g}$ are homogeneous of degree $g$. Consider $\operatorname{supp}(R)=\{g \in$ $\left.G \mid R_{g} \neq 0\right\}$. An element $r$ of $R$ has a unique decomposition as $r=\sum_{g \in G} r_{g}$ with $r_{g} \in R_{g}$ for all $g \in G$. Moreover, we put $h(R)=\bigcup_{g \in G} R_{g}$.

Definition 2.2. [14] Let $R=\bigoplus_{g \in G} R_{g}$ be a graded ring. A subring (An ideal) $S$ of $R$ is called a graded subring (graded ideal) of $R$ if $S=\sum_{g \in G}\left(R_{g} \cap S\right)$. Equivalently, $S$ is graded if for every element $x \in S$ all the homogeneous components of $x$ (as an element of $R$ ) are in $S$. Moreover, $R / S$ becomes a $G$-graded ring with $g$-component $(R / S)_{g}=\left(R_{g}+S\right) / S$ for $g \in G$ in case $S$ is a graded ideal of $R$.

Proposition 2.3. [14] Let $R=\bigoplus_{g \in G} R_{g}$ be a graded ring. Then $1 \in R_{e}$ and $R_{e}$ is a subring of $R$.

Definition 2.4. [3] A graded ring $R$ is called graded quasilocal ring if it has a unique graded maximal ideal.

Definition 2.5. [14] Let $R$ be a graded ring and $M$ be an $R$-module. We say that $M$ is a graded $R$-module, if there exists a family of subgroups $\left\{M_{g}\right\}_{g \in G}$ of $M$ such that

(1) $M=\bigoplus_{g \in G} M_{g}$,

(2) $R_{g} M_{h} \subseteq M_{g h}$ for all $g, h \in G$. 
The elements of $M_{g}$ are called homogeneous of degree $g$. Also, we consider $\operatorname{supp}(M)=$ $\left\{g \in G \mid M_{g} \neq 0\right\}$.

It is clear that $M_{g}$ is an $R_{e}$-submodule of $M$ for all $g \in G$. Moreover, we put $h(M)=\bigcup_{g \in G} M_{g}$.

Definition 2.6. [14] Let $N$ be an $R$-submodule of a graded $R$-module $M$. Then $N$ is said to be a graded $R$-submodule if $N=\bigoplus_{g \in G}\left(N \cap M_{g}\right)$, i.e., for $m \in N$, $m=\sum_{g \in G} m_{g}$, where $m_{g} \in N$ for all $g \in G$. Moreover, $M / N$ becomes a $G$-graded module with $g$-component $(M / N)_{g}=\left(M_{g}+N\right) / N$ for $g \in G$.

Definition 2.7. [4] A proper graded submodule $N$ of a graded $R$-module $M$ is said to be graded prime, if $r_{g} m_{h} \in N$ where $r_{g} \in h(R)$ and $m_{h} \in h(M)$, then $m_{h} \in N$ or $r_{g} \in(N: M)$. A graded $R$-module $M$ is called graded prime, if the zero graded submodule is graded prime in $M$.

Definition 2.8. [2] A proper graded submodule $N$ of $M$ is called graded 2absorbing, if $a_{g} b_{h} m_{k} \in N$ for some $a_{g}, b_{h} \in h(R)$ and $m_{k} \in h(M)$, then $a_{g} b_{h} \in$ $\left(N:_{R} M\right)$ or $a_{g} m_{k} \in N$ or $b_{h} m_{k} \in N$.

Definition 2.9. [4] A graded $R$-module $M$ is called graded finitely generated if $M=R m_{g_{1}}+R m_{g_{2}}+\cdots+R m_{g_{n}}$ for some $m_{g_{1}}, \ldots, m_{g_{n}} \in h(M)$.

We call $S \subseteq h(R)$ is a multiplicatively closed subset of $R$ if $(i) 0 \notin S$, (ii) $1 \in S$, and (iii) $s_{g} s_{g^{\prime}}^{\prime} \in S$ for all $s_{g}, s_{g^{\prime}}^{\prime} \in S$.

Definition 2.10. [14] Let $S \subseteq h(R)$ be a multiplicatively closed subset of $R$ and $M$ be a graded $R$-module. Then $S^{-1} M$ is a graded $S^{-1} R$-module with

$$
\left(S^{-1} M\right)_{g}=\left\{\frac{m}{s}:(\operatorname{deg} m)(\operatorname{deg} s)^{-1}=g\right\}
$$

and

$$
\left(S^{-1} R\right)_{g}=\left\{\frac{r}{s}:(\operatorname{deg} r)(\operatorname{deg} s)^{-1}=g\right\} .
$$

Definition 2.11. [13] Let $M=\bigoplus_{g \in G} M_{g}$ and $M^{\prime}=\bigoplus_{g \in G} M_{g}^{\prime}$ be two graded $R$-modules. A mapping $f$ from $M$ into $M^{\prime}$ is said to be a graded homomorphism, if for all $m, n \in M$;

(1) $f(m+n)=f(m)+f(n)$.

(2) $f(r m)=r f(m)$, for any $r \in R$ and $m \in M$.

(3) For any $g \in G ; f\left(M_{g}\right) \subseteq M_{g}^{\prime}$. 
Definition 2.12. [5] A graded $R$-module $M$ is called a graded multiplication module, if every graded submodule $N$ of $M, N=I M$ for some graded ideal $I$ of $R$. Since $I \subseteq\left(N:_{R} M\right), N=I M \subseteq\left(N:_{R} M\right) M \subseteq N$. Hence if $M$ is graded multiplication, $N=\left(N:_{R} M\right) M$, for every graded submodule $N$ of $M$.

Assume that $M$ is a graded multiplication $R$-module and $K, L$ are two graded submodules of $M$. Then $K=I_{1} M$ and $L=I_{2} M$ for some graded ideals $I_{1}, I_{2}$ of $R$. The product $K$ and $L$ denoted by $K L$ is defined by $K L=I_{1} I_{2} M$.

Definition 2.13. [5] Let $Q$ be a graded maximal ideal of $R$. Consider the graded submodule $T_{Q}(M)=\{m \in M \mid$ there is a $q \in h(R) \backslash Q$ such that $q m=0\}$ of $M$. If $T_{Q}(M)=M$, we call $M$ is a graded $Q$-torsion module. If there exist $q \in h(R) \backslash Q$ and $m \in h(M)$ such that $q M \subseteq R m$, we say $M$ is a graded $Q$-cyclic module.

Theorem 2.14. [5] $M$ is a graded multiplication $R$-module if and only if for every graded maximal ideal $Q$ of $R$, either $M$ is a graded $Q$-cyclic or $M$ is a graded $Q$-torsion module.

\section{Graded $S$-1-absorbing prime submodules}

Definition 3.1. Let $M$ be a graded $R$-module and $N$ a proper graded submodule of $M$. Then $N$ is said to be a graded 1-absorbing prime submodule if $a_{h} b_{h^{\prime}} m_{k} \in N$ implies that either $a_{h} b_{h^{\prime}} \in\left(N:_{R} M\right)$ or $m_{k} \in N$ for all non-units $a_{h}, b_{h^{\prime}} \in h(R)$ and all $m_{k} \in h(M)$.

It is clear that if $I$ is a graded 1-absorbing prime ideal of $R$, it is a graded 1-absorbing prime submodule of graded $R$-module $R$.

The following notion generalizes the notion from Definition 2.1 in [6].

Definition 3.2. Let $S \subseteq h(R)$ be a multiplicatively closed subset of a graded ring $R$ and $N$ be a graded submodule of $M$ with $\left(N:_{R} M\right) \cap S=\emptyset$. Then $N$ is said to be a graded $S$-1-absorbing prime submodule if there exists an $s_{g} \in S$ such that whenever $a_{h} b_{h^{\prime}} m_{k} \in N$, then either $s_{g} a_{h} b_{h^{\prime}} \in\left(N:_{R} M\right)$ or $s_{g} m_{k} \in N$ for all non-units $a_{h}, b_{h^{\prime}} \in h(R)$ and all $m_{k} \in h(M)$.

It is clear that if $I$ is a graded $S$-1-absorbing prime ideal of $R$, it is a graded $S$-1-absorbing prime submodule of graded $R$-module $R$.

Example 3.3. (i) Let $M$ be a graded $R$-module and $S \subseteq h(R)$ a multiplicatively closed subset of $R$. Every graded 1-absorbing prime submodule $N$ of $M$ with $\left(N:_{R} M\right) \cap S=\emptyset$ is also a graded $S$-1-absorbing prime submodule of $M$. 
(ii) Let $M$ be a graded $R$-module and $S \subseteq h(R)$ a multiplicatively closed subset of $R$ consisting of units in $R$. Then a graded submodule $N$ of $M$ is graded 1-absorbing prime if and only if $N$ is graded $S$-1-absorbing prime.

(iii) Let us observe $R=\mathbb{Z}$ as a trivially $\mathbb{Z}_{2}$-graded ring and $M=\mathbb{Z} \times \mathbb{Z}_{4}$ be a $\mathbb{Z}_{2}$-graded $R$-module with $M_{0}=\mathbb{Z} \times\{0\}$ and $M_{1}=\{0\} \times \mathbb{Z}_{4}$. Consider the zero graded submodule $N=0 \times 0$. Note that $\left(N: \mathbb{Z} \mathbb{Z} \times \mathbb{Z}_{4}\right)=0$ and $2^{2}(0, \overline{1}) \in N$ where $2 \in R_{0}$ and $(0, \overline{1}) \in M_{1}$. Since $4 \notin\left(N: \mathbb{Z} \mathbb{Z} \times \mathbb{Z}_{4}\right)$ and $(0, \overline{1}) \notin N, N$ is not a graded 1- absorbing prime submodule of $M=\mathbb{Z} \times \mathbb{Z}_{4}$. Now, take the multiplicatively closed subset $S=\mathbb{Z}-\{0\}$ and put $s=4$. It is easy to see that $N$ is a graded $S$-1-absorbing prime submodule of $\mathbb{Z} \times \mathbb{Z}_{4}$.

Proposition 3.4. Let $S \subseteq h(R)$ be a multiplicatively closed subset of $R$ and $M$ be a graded $R$-module. Then every graded $S$-prime submodule is a graded $S$-1absorbing prime submodule, and every graded $S$-1-absorbing prime submodule is a graded S-2-absorbing submodule.

Proof. Let $N$ be a graded $S$-prime submodule of $M$. Suppose that $a_{h} b_{h^{\prime}} m_{k} \in N$ for some non-unit elements $a_{h}, b_{h^{\prime}} \in h(R)$ and $m_{k} \in h(M)$. Since $N$ is a graded $S$-prime submodule of $M$, there exists an $s_{g} \in S$ so that $s_{g} a_{h} b_{h^{\prime}} \in\left(N:_{R} M\right)$ or $s_{g} m_{k} \in N$, as needed. Now let $N$ be a graded $S$-1-absorbing prime submodule. Let $a_{h} b_{h^{\prime}} m_{k} \in N$ where $a_{h}, b_{h^{\prime}} \in h(R)$ and $m_{k} \in h(M)$. If $a_{h}, b_{h^{\prime}}$ are non-unit homogeneous elements, since $N$ is a graded $S$-1-absorbing prime submodule, there exists an $s_{g} \in S$ so that $s_{g} a_{h} b_{h^{\prime}} \in\left(N:_{R} M\right)$ or $s_{g} m_{k} \in N$, it is done. Without loss generality, assume that $a_{h}$ is unit. Then $a_{h} b_{h^{\prime}} m_{k} \in N$, implies that $b_{h^{\prime}} m_{k} \in N$ and so $N$ is a graded $S$-2-absorbing submodule of $M$.

Example 3.5. Let $G=\mathbb{Z}_{2}$. Let $R=\mathbb{Z}_{4}$ be $G$-graded ring and $M=\mathbb{Z}_{4}[X]$ be a graded $R$-module with $M_{0}=\mathbb{Z}_{4}$ and $M_{1}=\langle X\rangle$. Consider the graded submodule $N=\langle X\rangle$. Take the multiplicatively closed subset $S=\mathbb{Z}_{4}-\langle\overline{2}\rangle=\{\overline{1}, \overline{3}\}$ of $\mathbb{Z}_{4}$. Then $N$ is a graded $S$-1-absorbing prime submodule of $\mathbb{Z}_{4}[X]$, but $N$ is not a graded $S$-prime submodule of $\mathbb{Z}_{4}[X]$.

Let $S \subseteq h(R)$ be a multiplicatively closed subset of $R$. The saturation $S^{*}$ of $S$ is defined as $S^{*}=\left\{x \in h(R) \mid \frac{x}{1}\right.$ is a unit of $\left.S^{-1} R\right\}$. Note that $S^{*}$ is a multiplicatively closed subset containing $S$.

The following proposition represents a generalization of Proposition 2.2 in [6].

Proposition 3.6. Let $M$ be a graded $R$-module and $S \subseteq h(R)$ be a multiplicatively closed subset of $R$. Then the following statements hold: 
(i) Let $S_{1} \subseteq S_{2} \subseteq h(R)$ be multiplicatively closed subsets of $R$. If $N$ is a graded $S_{1}$-1-absorbing prime submodule and $\left(N:_{R} M\right) \cap S_{2}=\emptyset$, then $N$ is a graded $S_{2}$-1-absorbing prime submodule.

(ii) A graded submodule $N$ of $M$ is a graded $S$-1-absorbing prime submodule if and only if it is a graded $S^{*}$-1-absorbing prime submodule.

(iii) If $N$ is a graded $S$-1-absorbing prime submodule of $M$, then $S^{-1} N$ is a graded 1-absorbing prime submodule of $S^{-1} M$.

Proof. (i) It is clear.

(ii) Let $N$ be a graded $S$-1-absorbing prime submodule. Assume that $\left(N:_{R}\right.$ $M) \cap S^{*} \neq \emptyset$ and let $r_{g} \in\left(N:_{R} M\right) \cap S^{*}$. Hence $\frac{r_{g}}{1}$ is a unit homogeneous element of $S^{-1} R$, that is, $\frac{r_{g}}{1} \frac{a_{h}}{s_{g^{\prime}}}=\frac{1}{1}$ for some $a_{h} \in h(R)$ and $s_{g^{\prime}} \in S$. Thus $u_{g^{\prime \prime}} s_{g^{\prime}}=u_{g^{\prime \prime}} r_{g} a_{h} \in S$ for some $u_{g^{\prime \prime}} \in S$. Then $u_{g^{\prime \prime}} s_{g^{\prime}}=u_{g^{\prime \prime}} r_{g} a_{h} \in\left(N:_{R} M\right) \cap S$, which is a contradiction. Thus $\left(N:_{R} M\right) \cap S^{*}=\emptyset$. Since $S \subseteq S^{*}$, by $(i), N$ is a graded $S^{*}$-1-absorbing prime submodule of $M$. Conversely, assume that $N$ is a graded $S^{*}$-1-absorbing prime submodule. Let $a_{h} b_{h} m_{k} \in N$ for some non-unit elements $a_{h}, b_{h^{\prime}} \in h(R)$ and $m_{k} \in h(M)$. Since $N$ is a graded $S^{*}$-1-absorbing prime submodule, there is an $s_{g}^{\prime} \in S^{*}$ so that either $s_{g}^{\prime} a_{h} b_{h^{\prime}} \in\left(N:_{R} M\right)$ or $s_{g}^{\prime} m_{k} \in N$. As $\frac{s_{g}^{\prime}}{1}$ is a unit of $S^{-1} R$, there exist $u_{g^{\prime}}, s_{g^{\prime \prime}} \in S$ and $r_{h^{\prime \prime}} \in h(R)$ such that $s_{g^{\prime \prime}} u_{g^{\prime}}=$ $u_{g^{\prime}} s_{g}^{\prime} r_{h^{\prime \prime}}$. Put $u_{g^{\prime}} s_{g}^{\prime}=s_{g^{\prime} g}^{\prime \prime} \in S$. Then note that $s_{g^{\prime} g}^{\prime \prime} a_{h} b_{h^{\prime}}=\left(u_{g^{\prime}} s_{g^{\prime \prime}}\right) a_{h} b_{h^{\prime}}=$ $u_{g^{\prime}} r_{h^{\prime \prime}} s_{g}^{\prime} a_{h} b_{h^{\prime}} \in\left(N:_{R} M\right)$ or $s_{g^{\prime} g}^{\prime \prime} m_{k}=u_{g^{\prime}} r_{h^{\prime \prime}}\left(s_{g}^{\prime} m_{k}\right) \in N$. Therefore, $N$ is a graded $S$-1-absorbing prime submodule.

(iii) Let $N$ be a graded $S$-1-absorbing prime submodule of $M$ and $\frac{a_{h}}{s_{g}} \frac{b_{h^{\prime}}}{t_{g^{\prime}}} \frac{m_{k}}{u_{g^{\prime \prime}}} \in$ $S^{-1} N$, for some non-unit elements $\frac{a_{h}}{s_{g}}, \frac{b_{h^{\prime}}}{t_{g^{\prime}}} \in h\left(S^{-1} R\right)$ and $\frac{m_{k}}{u_{g^{\prime \prime}}} \in h\left(S^{-1} M\right)$. Then $s_{g^{\prime \prime \prime}}^{\prime} a_{h} b_{h^{\prime}} m_{k} \in N$ for some $s_{g^{\prime \prime \prime}}^{\prime} \in S$. Note that $a_{h}, b_{h^{\prime}}$ are non-unit homogeneous elements of $R$, because if $a_{h} a_{h^{-1}}^{\prime}=a_{h^{-1}}^{\prime} a_{h}=1$ for some $a_{h^{-1}}^{\prime} \in h(R)$, then

$$
\frac{a_{h}}{s_{g}} \frac{a_{h^{-1}}^{\prime} s_{g}}{1}=\frac{1}{1}
$$

a contradiction. Since $N$ is a graded $S$-1-absorbing prime submodule, there exists an $s_{k^{\prime}}^{\prime \prime} \in S$ so that $s_{k^{\prime}}^{\prime \prime} a_{h} b_{h^{\prime}} \in\left(N:_{R} M\right)$ or $s_{g^{\prime \prime \prime}}^{\prime \prime} s_{k^{\prime}}^{\prime \prime} m_{k} \in N$. Hence we have

$$
\frac{a_{h} b_{h^{\prime}}}{s_{g} t_{g^{\prime}}}=\frac{s_{k^{\prime}}^{\prime \prime} a_{h} b_{h^{\prime}}}{s_{k^{\prime}}^{\prime \prime} s_{g} t_{g^{\prime}}} \in S^{-1}\left(N:_{R} M\right) \subseteq\left(S^{-1} N:_{S^{-1} R} S^{-1} M\right)
$$

or $\frac{m_{k}}{u_{g^{\prime \prime}}}=\frac{s_{g^{\prime \prime}}^{\prime} s_{k^{\prime}}^{\prime \prime} m_{k}}{s_{g^{\prime \prime \prime}}^{\prime} s_{k^{\prime}}^{\prime \prime} u_{g^{\prime \prime}}} \in S^{-1} N$. Therefore, $S^{-1} N$ is a graded 1-absorbing prime submodule of $S^{-1} M$. 
Example 3.7. Consider the $\mathbb{Z}_{2}$-graded $\mathbb{Z}$-module $\mathbb{Q} \times \mathbb{Q}$ with $M_{0}=\mathbb{Q} \times\{0\}$ and $M_{1}=\{0\} \times \mathbb{Q}$, where $\mathbb{Q}$ is the field of rational numbers. Take the multiplicatively closed subset $S=\mathbb{Z}-\{0\}$ of $\mathbb{Z}$ and the graded submodule $N=\mathbb{Z} \times\{0\}$. It is clear that $(N: \mathbb{Z} \mathbb{Q} \times \mathbb{Q})=0$. Let $s$ be an arbitrary element of $S$. Choose a prime number $p$ with $\operatorname{gcd}(p, s)=1$. Then $p^{2}\left(\frac{1}{p}, 0\right)=(p, 0) \in N$. Since $s p^{2} \notin(N: \mathbb{Z} \mathbb{Q} \times \mathbb{Q})$ and $s\left(\frac{1}{p}, 0\right)=\left(\frac{s}{p}, 0\right) \notin N$, it follows that $N$ is not a graded $S$-1-absorbing prime submodule. Since $S^{-1} \mathbb{Z}=\mathbb{Q}$ is a field, $S^{-1}(\mathbb{Q} \times \mathbb{Q})$ is a vector space so that the proper graded submodule $S^{-1} N$ is a graded 1-absorbing prime submodule of $S^{-1}(\mathbb{Q} \times \mathbb{Q})$.

Proposition 3.8. Let $S \subseteq h(R)$ be a multiplicatively closed subset of $R$ and $M$ be a graded $R$-module. Let $N$ be a graded $S$-1-absorbing prime submodule of $M$. Then

(i) $\left(N:_{R} M\right)$ is a graded $S$-1-absorbing prime ideal of $R$.

(ii) if $\left(N:_{R} m_{g}\right) \cap S=\emptyset$ for each $m_{g} \in h(M)$, then $\left(N:_{R} m_{g}\right)$ is a graded $S$-1-absorbing prime ideal of $R$.

Proof. (i) Take non-units $a_{h}, b_{h^{\prime}}, c_{h^{\prime \prime \prime}} \in h(R)$ such that $a_{h} b_{h^{\prime}} c_{h^{\prime \prime}} \in\left(N:_{R} M\right)$. Then $a_{h} b_{h^{\prime}} c_{h^{\prime \prime}} m \in N$ for any $m \in M$. We can write $m=\sum_{g \in G} m_{g}$ where $m_{g} \in M_{g}$. Then for any $g \in G, a_{h} b_{h^{\prime}} c_{h^{\prime \prime}} m_{g} \in N$. Since $N$ is a graded $S$-1-absorbing prime submodule of $M$, there exists an $s_{g^{\prime}} \in S$ such that $s_{g^{\prime}} a_{h} b_{h^{\prime}} \in\left(N:_{R} M\right)$ or $s_{g^{\prime}} c_{h^{\prime \prime}} m_{g} \in N$. Therefore, $s_{g^{\prime}} a_{h} b_{h^{\prime \prime}} \in\left(N:_{R} M\right)$ or $s_{g^{\prime}} c_{h^{\prime \prime}} \in\left(N:_{R} M\right)$, as required.

(ii) It follows from (i).

The converse of Proposition 3.8 is not true for general cases. Consider the following example.

Example 3.9. Consider the $\mathbb{Z}_{2}$-graded $\mathbb{Z}$-module $\mathbb{Z} \times \mathbb{Z}$ with $M_{0}=\mathbb{Z} \times\{0\}$ and $M_{1}=\{0\} \times \mathbb{Z}$ and the graded submodule $N=\langle(3,0)\rangle$. Take the multiplicatively closed subset $S=\mathbb{Z}-3 \mathbb{Z}$ of $\mathbb{Z}$. It is clear that $(N: \mathbb{Z} \mathbb{Z} \times \mathbb{Z})=0$ is a graded $S$-1-absorbing prime ideal of $\mathbb{Z}$. Let $s$ be an arbitrary element of $S$. Choose nonunit elements $2,3 \in h(\mathbb{Z})=\mathbb{Z}$ and $(1,0) \in h(\mathbb{Z} \times \mathbb{Z})$, then $2 \cdot 3 \cdot(1,0) \in N$. But $s 6 \notin\left(N:_{\mathbb{Z}} \mathbb{Z} \times \mathbb{Z}\right)=0$ and $s(1,0) \notin N$. Therefore, $N$ is not a graded $S$-1-absorbing prime submodule of $\mathbb{Z} \times \mathbb{Z}$.

Lemma 3.10. Let $M$ be a graded $R$-module, $S \subseteq h(R)$ be a multiplicatively closed subset of $R$ and $N$ be a graded $S$-1-absorbing prime submodule of $M$. Then there exists an $s_{g} \in S$ such that whenever $a_{h} b_{h^{\prime}} K \subseteq N$, then either $s_{g} a_{h} b_{h^{\prime}} \in\left(N:_{R} M\right)$ or $s_{g} K \subseteq N$ for all non-unit elements $a_{h}, b_{h^{\prime}} \in h(R)$ and all graded submodules $K$ of $M$. 
Proof. Assume that $a_{h} b_{h^{\prime}} K \subseteq N$ for some non-unit elements $a_{h}, b_{h^{\prime}} \in h(R)$ and a graded submodule $K$ of $M$. Since $N$ is a graded $S$-1-absorbing prime submodule of $M$, there exists an $s_{g} \in S$ such that whenever $a_{h} b_{h^{\prime}} m_{k} \in N$, then either $s_{g} a_{h} b_{h^{\prime}} \in$ $\left(N:_{R} M\right)$ or $s_{g} m_{k} \in N$ for all non-units $a_{h}, b_{h^{\prime}} \in h(R)$ and all $m_{k} \in h(M)$. Suppose that $s_{g} K \nsubseteq N$. Then there is $x_{k} \in K$ such that $s_{g} x_{k} \notin N$. We have $a_{h} b_{h^{\prime}} x_{k} \in N$, thus $s_{g} a_{h} b_{h^{\prime}} \in\left(N:_{R} M\right)$ or $s_{g} x_{k} \in N$ since $N$ is a graded $S$-1absorbing prime submodule. The second one implies a contradiction, we conclude $s_{g} a_{h} b_{h^{\prime}} \in\left(N:_{R} M\right)$, as required.

The following theorem represents a generalization of Theorem 2.1 in [6].

Theorem 3.11. Let $M$ be a graded $R$-module and $S \subseteq h(R)$ a multiplicatively closed subset of $R$, and $N$ a graded submodule of $M$ such that $\left(N:_{R} M\right) \cap S=\emptyset$. Then the following statements are equivalent:

(i) $N$ is a graded $S$-1-absorbing prime submodule of $M$.

(ii) There exists an $s_{g} \in S$ such that whenever $I J K \subseteq N$, then either $s_{g} I J \subseteq$ $\left(N:_{R} M\right)$ or $s_{g} K \subseteq N$ for all graded ideals $I, J$ of $R$ and all graded submodules $K$ of $M$.

Proof. (i) $\Rightarrow$ (ii) Suppose that $N$ is a graded $S$-1-absorbing prime submodule of $M$. Let $I J K \subseteq N$ for some graded ideals $I, J$ of $R$ and a graded submodule $K$ of $M$. Since $N$ is graded $S$-1-absorbing prime, there exists an $s_{g} \in S$ such that whenever $a_{h} b_{h^{\prime}} m_{k} \in N$ implies that either $s_{g} a_{h} b_{h^{\prime}} \in\left(N:_{R} M\right)$ or $s_{g} m_{k} \in N$ for all non-unit elements $a_{h}, b_{h^{\prime}} \in h(R)$ and all $m_{k} \in h(M)$. Assume that $s_{g} I J \nsubseteq$ $\left(N:_{R} M\right)$. Then there are non-unit elements $a_{h} \in I \cap h(R)$ and $b_{h^{\prime}} \in J \cap h(R)$ and $s_{g} a_{h} b_{h^{\prime}} \notin\left(N:_{R} M\right)$. As $a_{h} b_{h^{\prime}} K \subseteq N$, we conclude $s_{g} K \subseteq N$ by Lemma 3.10.

(ii) $\Rightarrow$ (i) Let $a_{h} b_{h^{\prime}} m_{k} \in N$ where $a_{h}, b_{h^{\prime}}$ are non-unit elements of $R$ and $m_{k} \in$ $h(M)$. Take $I=R a_{h}, J=R b_{h^{\prime}}$ and $K=R m_{k}$. Then $I J K=R a_{h} R b_{h^{\prime}} R m_{k} \subseteq N$, so $s_{g} I J \subseteq\left(N:_{R} M\right)$ or $s_{g} K \subseteq N$ by hypothesis, hence $s_{g} a_{h} b_{h^{\prime}} \in\left(N:_{R} M\right)$ or $s_{g} m_{k} \in N$. Thus $N$ is a graded $S$-1-absorbing prime submodule of $M$.

Corollary 3.12. Let $S \subseteq h(R)$ be a multiplicatively closed subset of $R$ and $P$ a graded ideal of $R$ with $P \cap S=\emptyset$. Then $P$ is a graded $S$-1-absorbing prime ideal of $R$ if and only if there is an $s_{g} \in S$, for any graded ideal $I, J, K$ of $R$ with $I J K \subseteq P$, then $s_{g} I J \subseteq P$ or $s_{g} K \subseteq P$.

Proposition 3.13. Let $S \subseteq h(R)$ be a multiplicatively closed subset of $R$ and $f: M \rightarrow M^{\prime}$ be a graded R-homomorphism. Then the following hold: 
(i) If $N^{\prime}$ is a graded $S$-1-absorbing prime submodule of $M^{\prime}$ such that $\left(f^{-1}\left(N^{\prime}\right):_{R}\right.$ $M) \cap S=\emptyset$, then $f^{-1}\left(N^{\prime}\right)$ is a graded $S$-1-absorbing prime submodule of $M$.

(ii) If $f$ is a graded epimorphism and $N$ is a graded $S$-1-absorbing prime submodule of $M$ containing $\operatorname{Ker}(f)$, then $f(N)$ is a graded $S$-1-absorbing prime submodule of $M^{\prime}$.

Proof. (i) Let $a_{h} b_{h^{\prime}} m_{k} \in f^{-1}\left(N^{\prime}\right)$ for some non-unit elements $a_{h}, b_{h^{\prime}} \in h(R)$ and $m_{k} \in h(M)$. Hence $f\left(a_{h} b_{h^{\prime}} m_{k}\right)=a_{h} b_{h^{\prime}} f\left(m_{k}\right) \in N^{\prime}$. Since $N^{\prime}$ is a graded $S$ 1-absorbing prime submodule of $M^{\prime}$, there exists an $s_{g} \in S$ such that $s_{g} a_{h} b_{h^{\prime}} \in$ $\left(N^{\prime}:_{R} M^{\prime}\right)$ or $s_{g} f\left(m_{k}\right)=f\left(s_{g} m_{k}\right) \in N^{\prime}$. We have $\left(N^{\prime}:_{R} M^{\prime}\right) \subseteq\left(f^{-1}\left(N^{\prime}\right):_{R} M\right)$, and so $s_{g} a_{h} b_{h^{\prime}} \in\left(f^{-1}\left(N^{\prime}\right):_{R} M\right)$ or $s_{g} m_{k} \in f^{-1}\left(N^{\prime}\right)$. Thus $f^{-1}\left(N^{\prime}\right)$ is a graded $S$-1-absorbing prime submodule of $M$.

(ii) We have $\left(f(N):_{R} M^{\prime}\right) \cap S=\emptyset$, because if $s_{g} \in\left(f(N):_{R} M^{\prime}\right) \cap S$, then $s_{g} \in\left(f(N):_{R} M^{\prime}\right), s_{g} M^{\prime} \subseteq f(N)$, and so $f\left(s_{g} M\right)=s_{g} f(M) \subseteq f(N)$, thus we have $s_{g} M \subseteq s_{g} M+\operatorname{Ker}(f) \subseteq N+\operatorname{Ker}(f)=N$, a contradiction. Take non-units $a_{h}, b_{h^{\prime}} \in h(R)$ and $m_{k}^{\prime} \in h\left(M^{\prime}\right)$ such that $a_{h} b_{h^{\prime}} m_{k}^{\prime} \in f(N)$. Since $f$ is a graded epimorphism, there is an $m_{k} \in h(M)$ such that $m_{k}^{\prime}=f\left(m_{k}\right)$. Then $a_{h} b_{h^{\prime}} m_{k}^{\prime}=$ $a_{h} b_{h^{\prime}} f\left(m_{k}\right)=f\left(a_{h} b_{h^{\prime}} m_{k}\right) \in f(N)$, so $a_{h} b_{h^{\prime}} m_{k} \in f^{-1}(f(N)) \subseteq N$ since $\operatorname{Ker}(f) \subseteq$ $N$. Since $N$ is a graded $S$-1-absorbing prime submodule of $M$, there exists an $s_{g} \in S$ so that $s_{g} a_{h} b_{h^{\prime}} \in\left(N:_{R} M\right)$ or $s_{g} m_{k} \in N$. As $\left(N:_{R} M\right) \subseteq\left(f(N):_{R} M^{\prime}\right)$, we have $s_{g} a_{h} b_{h^{\prime}} \in\left(f(N):_{R} M^{\prime}\right)$ or $f\left(s_{g} m_{k}\right)=s_{g} f\left(m_{k}\right)=s_{g} m_{k}^{\prime} \in f(N)$. Therefore, $f(N)$ is a graded $S$-1-absorbing prime submodule of $M^{\prime}$.

Corollary 3.14. Let $S \subseteq h(R)$ be a multiplicatively closed subset of $R$ and $N, K$ be graded submodules of $M$ with $K \subseteq N$. Then the following hold:

(i) If $N^{\prime}$ is a graded $S$-1-absorbing prime submodule of $M$ with $\left(N^{\prime}:_{R} K\right) \cap S=$ $\emptyset$, then $K \cap N^{\prime}$ is a graded $S$-1-absorbing prime submodule of $K$.

(ii) If $N$ is a graded $S$-1-absorbing prime submodule of $M$ if and only if $N / K$ is a graded $S$-1-absorbing prime submodule of $M / K$.

Proof. (i) Consider the injection $i: K \rightarrow M$ defined by $i(x)=x$ for all $x \in K$. Then $i^{-1}\left(N^{\prime}\right)=K \cap N^{\prime}$. By $\left(N^{\prime}:_{R} K\right) \cap S=\emptyset$, we get $\left(i^{-1}\left(N^{\prime}\right):_{R} K\right) \cap S=\emptyset$. Thus the rest follows from Proposition 3.13(i).

(ii) Let $N$ be a graded $S$-1-absorbing prime submodule of $M$. Then consider the canonical homomorphism $\pi: M \rightarrow M / K$ defined by $\pi(m)=m+K$ for all $m \in M$. Then note that $\pi$ is an epimorphism and $\operatorname{Ker}(\pi)=K \subseteq N$. Thus by Proposition 3.13(ii), $N / K$ is a graded $S$-1-absorbing prime submodule of $M / K$. 
Conversely, assume that $N / K$ is a graded $S$-1-absorbing prime submodule of $M / K$. Let $a_{h} b_{h^{\prime}} m_{k} \in N$ for some non-units $a_{h}, b_{h^{\prime}} \in h(R)$ and $m_{k} \in h(M)$. Then $a_{h} b_{h^{\prime}}\left(m_{k}+K\right) \in N / K$. Since $N / K$ is a graded $S$-1-absorbing prime submodule of $M / K$, there is an $s_{g} \in S$ so that $s_{g}\left(m_{k}+K\right) \in N / K$ or $s_{g} a_{h} b_{h^{\prime}} \in\left(N / K:_{R}\right.$ $M / K)=\left(N:_{R} M\right)$, and so $s_{g} m_{k} \in N$ or $s_{g} a_{h} b_{h^{\prime}} \in\left(N:_{R} M\right)$, as needed.

Let $R_{1}$ and $R_{2}$ be $G$-graded rings. Then $R=R_{1} \times R_{2}$ is a $G$-graded ring with $R_{g}=\left(R_{1}\right)_{g} \times\left(R_{2}\right)_{g}$ for all $g \in G$. Let $M_{1}$ be a $G$-graded $R_{1}$-module, $M_{2}$ be a $G$ graded $R_{2}$-module and $R=R_{1} \times R_{2}$. Then $M=M_{1} \times M_{2}$ is a $G$-graded $R$-module with $M_{g}=\left(M_{1}\right)_{g} \times\left(M_{2}\right)_{g}$ for all $g \in G$. Also, if $S_{1} \subseteq h\left(R_{1}\right)$ is a multiplicatively closed subset of $R_{1}$ and $S_{2} \subseteq h\left(R_{2}\right)$ is a multiplicatively closed subset of $R_{2}$, then $S=S_{1} \times S_{2}$ is a multiplicatively closed subset of $R$. Furthermore, each graded submodule of $M$ is of the form $N=N_{1} \times N_{2}$ where $N_{i}$ is a graded submodule of $M_{i}$ for $i=1,2$.

The following theorem represents a generalization of Theorem 2.2 in [6].

Theorem 3.15. Let $M=M_{1} \times M_{2}$ be a graded $R=R_{1} \times R_{2}$-module and $S=$ $S_{1} \times S_{2}$ be a multiplicatively closed subset of $R$ where $M_{i}$ is a graded $R_{i}$-module and $S_{i} \subseteq h\left(R_{i}\right)$ is a multiplicatively closed subset of $R_{i}$, for each $=1,2$. Suppose that $N_{1}$ is a graded submodule of $M_{1}$ and $N_{2}$ is a graded submodule of $M_{2}$ and $N=N_{1} \times N_{2}$. Then if $N$ is a graded $S$-1-absorbing prime submodule of $M$, then $\left(N_{1}: R_{1} M_{1}\right) \cap S_{1} \neq \emptyset$ and $N_{2}$ is a graded $S_{2}$-1-absorbing prime submodule of $M_{2}$ or $\left(N_{2}:_{R_{2}} M_{2}\right) \cap S_{2} \neq \emptyset$ and $N_{1}$ is a graded $S_{1}$-1-absorbing prime submodule of $M_{1}$.

Proof. Assume that $N$ is a graded $S$-1-absorbing prime submodule of $M$. First, note that $\left(N:_{R} M\right)=\left(N_{1}:_{R_{1}} M_{1}\right) \times\left(N_{2}:_{R_{2}} M_{2}\right)$ is a graded $S$-1-absorbing prime ideal of $R$ by Proposition 3.8(i). Hence $\left(N_{1}:_{R} M_{1}\right) \cap S_{1}=\emptyset$ or $\left(N_{2}:_{R} M_{2}\right) \cap S_{2}=\emptyset$. Suppose that $\left(N_{1}:_{R} M_{1}\right) \cap S_{1} \neq \emptyset$. We will show that $N_{2}$ is a graded $S$-1-absorbing prime submodule of $M_{2}$. Let $a_{h} b_{h^{\prime}} m_{k} \in N_{2}$ where $a_{h}, b_{h^{\prime}}$ are non-unit elements of $R_{2}$ and $m_{k} \in M_{2}$. Then $\left(0, a_{h}\right)\left(0, b_{h^{\prime}}\right)\left(0, m_{k}\right) \in N_{1} \times N_{2}=N$. As $N=N_{1} \times N_{2}$ is a graded $S$-1-absorbing prime submodule of $M$, there exists an $s_{g}=\left(s_{g}^{\prime}, s_{g}^{\prime \prime}\right) \in S$ such that $s_{g}\left(0, m_{k}\right)=\left(0, s_{g}^{\prime \prime} m_{k}\right) \in N$ or $s_{g}\left(0, a_{h}\right)\left(0, b_{h^{\prime}}\right)=\left(0, s_{g}^{\prime \prime} a_{h} b_{h^{\prime}}\right) \in\left(N:_{R} M\right)$. Thus $s_{g}^{\prime \prime} m_{k} \in N_{2}$ or $s_{g}^{\prime \prime} a_{h} b_{h^{\prime}} \in\left(N_{2}:_{R_{2}} M_{2}\right)$. Hence $N_{2}$ is a graded $S_{2}$-1-absorbing prime submodule of $M_{2}$. If $\left(N_{2}:_{R_{2}} M_{2}\right) \cap S_{2} \neq \emptyset$, similarly $N_{1}$ is a graded $S_{1}-1$ absorbing prime submodule of $M_{1}$.

Assume that $M$ be a $G$-graded $R$-module. The idealization $R(+) M=\{(a, m) \mid a \in$ $R, m \in M\}$ of $M$ is a commutative ring whose addition is componentwise and whose multiplication is defined as $(a, m)\left(b, m^{\prime}\right)=\left(a b, a m^{\prime}+b m\right)$ for each $a, b \in R$ 
and $m, m^{\prime} \in M$. Also, it is a $G$-graded ring with $(R(+) M)_{g}=R_{g}(+) M_{g}$ for all $g \in G$. Let $I$ be a graded ideal of $R$ and $N$ a graded submodule of $M$. Then $I(+) N$ is a graded ideal of $R(+) M$ if and only if $I M \subseteq N$. Also, If $S \subseteq h(R)$ is a multiplicatively closed subset of $R$ and $N$ is a graded submodule of $M$, then $S(+) N=\{(s, n) \mid s \in S, n \in N \cap h(M)\}$ is a multiplicatively closed subset of $R(+) M$.

Proposition 3.16. Let $S \subseteq h(R)$ be a multiplicatively closed subset of $R$ and $I$ be a graded ideal of $R$ with $I \cap S=\emptyset$. Then the following statements are equivalent:

(i) $I$ is a graded $S$-1-absorbing prime ideal of $R$.

(ii) $I(+) M$ is a graded $S(+) 0$-1-absorbing prime ideal of $R(+) M$.

(iii) $I(+) M$ is a graded $S(+) h(M)$-1-absorbing prime ideal of $R(+) M$.

Proof. (i) $\Rightarrow$ (ii) Assume that $\left(a_{g}, m_{g}\right)\left(b_{h}, m_{h}^{\prime}\right)\left(c_{k}, m_{k}^{\prime \prime}\right)=\left(a_{g} b_{h} c_{k}, a_{g} b_{h} m_{k}^{\prime \prime}+a_{g} c_{k} m_{h}^{\prime}+\right.$ $\left.c_{k} b_{h} m_{g}\right) \in I(+) M$ for some non-units $\left(a_{g}, m_{g}\right),\left(b_{h}, m_{h}^{\prime}\right),\left(c_{k}, m_{k}^{\prime \prime}\right) \in h(R(+) M)$. Then $a_{g} b_{h} c_{k} \in I$. We may assume that $a_{g}, b_{h}, c_{k}$ are non-unit elements of $R$. Without loss generality, let $a_{g}$ is unit. Then $a_{g^{-1}}^{\prime} a_{g}=a_{g} a_{g^{-1}}^{\prime}=1$ for some $a_{g^{-1}}^{\prime} \in h(R)$, so $\left(a_{g}, m_{g}\right)\left(a_{g^{-1}}^{\prime},\left(-a_{g^{-1}}^{\prime}\right)^{2} m_{g}\right)=(1,0)$, a contradiction. Since $I$ is a graded $S$-1-absorbing prime ideal, there exists an $s_{g^{\prime}} \in S$ such that $s_{g^{\prime}} a_{g} b_{h} \in I$ or $s_{g^{\prime}} c_{k} \in I$. Thus $\left(s_{g^{\prime}}, 0\right)\left(a_{g}, m_{g}\right)\left(b_{h}, m_{h}^{\prime}\right)=\left(s_{g^{\prime}} a_{g} b_{h}, s_{g^{\prime}} a_{g} m_{h}^{\prime}+s_{g^{\prime}} b_{h} m_{g}\right) \in I(+) M$ or $\left(s_{g^{\prime}}, 0\right)\left(c_{k}, m_{k}^{\prime \prime}\right)=\left(s_{g^{\prime}} c_{k}, s_{g^{\prime}} m_{k}^{\prime \prime}\right) \in I(+) M$ where $\left(s_{g^{\prime}}, 0\right) \in S(+) 0$. Therefore, $I(+) M$ is a graded $S(+) 0$-1-absorbing prime ideal of $R(+) M$.

(ii) $\Rightarrow$ (iii) It follows from Proposition 3.6 since $S(+) 0 \subseteq S(+) M$.

(iii) $\Rightarrow$ (i) Assume that $a_{g} b_{h} c_{k} \in I$ for some non-unit elements $a_{g}, b_{h}, c_{k} \in h(R)$. Then $\left(a_{g}, 0\right)\left(b_{h}, 0\right)\left(c_{k}, 0\right) \in I(+) M$. It is easy to see that $\left(a_{g}, 0\right),\left(b_{h}, 0\right),\left(c_{k}, 0\right)$ are non-unit elements of $h(R(+) M)$. Since $I(+) M$ is a graded $S(+) h(M)$-1-absorbing prime ideal of $R(+) M$, there exists an $\left(s_{g^{\prime}}, m_{g^{\prime}}\right) \in S(+) M$ such that

$$
\left(s_{g^{\prime}}, m_{g^{\prime}}\right)\left(a_{g}, 0\right)\left(b_{h}, 0\right)=\left(s_{g^{\prime}} a_{g} b_{h}, a_{g} b_{h} m_{g^{\prime}}\right) \in I(+) M
$$

or $\left(s_{g^{\prime}}, m_{g^{\prime}}\right)\left(c_{k}, 0\right)=\left(s_{g^{\prime}} c_{k}, c_{k} m_{g^{\prime}}\right) \in I(+) M$, and so $s_{g^{\prime}} a_{g} b_{h} \in I$ or $s_{g^{\prime}} c_{k} \in I$. Thus $I$ is a graded $S$-1-absorbing prime ideal of $R$.

Lemma 3.17. Let $S \subseteq h(R)$ be a multiplicatively closed subset of $R$ and $N$ be a graded $S$-1-absorbing prime submodule of a graded $R$-module $M$. The following statements hold for some $s_{g} \in S$ :

(i) $\left(N:_{M} s_{g^{\prime}}^{\prime 2}\right) \subseteq\left(N:_{M} s_{g}\right)$ for all $s_{g^{\prime}}^{\prime} \in S$.

(ii) $\left(\left(N:_{R} M\right):_{R} s_{g^{\prime}}^{\prime 2} \subseteq\left(\left(N:_{R} M\right):_{R} s_{g}\right)\right.$ for all $s_{g^{\prime}}^{\prime} \in S$. 
Proof. (i) Suppose that $N$ is a graded $S$-1-absorbing prime submodule of $M$. Then there exists an $s_{g} \in S$ such that whenever $a_{h} b_{h} m_{k} \in N$, implies that either $s_{g} a_{h} b_{h^{\prime}} \in\left(N:_{R} M\right)$ or $s_{g} m_{k} \in N$ for all non-units $a_{h}, b_{h^{\prime}} \in h(R)$ and all $m_{k} \in$ $h(M)$. Let $m \in\left(N:_{M} s_{g^{\prime}}^{\prime 2}\right)$, where $s_{g^{\prime}}^{\prime} \in S$. Then $m=\sum_{k \in G} m_{k}$ where $m_{k} \in h(M)$. Thus $s_{g^{\prime}}^{\prime 2} m_{k} \in N$ for any $k \in G$. If $s_{g^{\prime}}^{\prime}$ is unit, then $m_{k} \in N$, we are done. Let $s_{g^{\prime}}^{\prime}$ is non-unit. Since $N$ is a graded $S$-1-absorbing prime submodule, we conclude $s_{g} s_{g^{\prime}}^{\prime 2} \in\left(N:_{R} M\right)$ or $s_{g} m_{k} \in N$. Since $\left(N:_{R} M\right) \cap S=\emptyset$, we get $s_{g} m_{k} \in N$ for any $k \in G$, hence $m \in\left(N:_{M} s_{g}\right)$, as required.

(ii) It follows from (i).

The following theorem represents a generalization of Theorem 2.3 in [6].

Theorem 3.18. Let $S \subseteq h(R)$ be a multiplicatively closed subset of $R$ and $N$ be a graded submodule of a graded $R$-module $M$ provided $\left(N:_{R} M\right) \cap S=\emptyset$. Then the following are equivalent:

(i) $N$ is a graded $S$-1-absorbing prime submodule of $M$.

(ii) $\left(N:_{M} s_{g}\right)$ is a graded 1-absorbing prime submodule of $M$ for some $s_{g} \in S$.

Proof. (i) $\Rightarrow$ (ii) Since $N$ is graded $S$-1-absorbing prime, there exists an $s_{g} \in S$ so that $a_{h} b_{h^{\prime}} m_{k} \in N$ implies that $s_{g} a_{h} b_{h^{\prime}} \in\left(N:_{R} M\right)$ or $s_{g} m_{k} \in N$ for all nonunit elements $a_{h}, b_{h^{\prime}} \in h(R)$ and all $m_{k} \in h(M)$. Let $a_{h} b_{h^{\prime}} m_{k} \in\left(N:_{M} s_{g}\right)$ for some non-units $a_{h}, b_{h^{\prime}} \in h(R)$ and $m_{k} \in h(M)$. Then $a_{h} b_{h^{\prime}} s_{g} m_{k} \in N$. We get $s_{g}^{2} a_{h} b_{h^{\prime}} \in\left(N:_{R} M\right)$ or $s_{g} m_{k} \in N$. If $s_{g} m_{k} \in N$, we are done. Assume that $s_{g}^{2} a_{h} b_{h^{\prime}} \in\left(N:_{R} M\right)$, then $a_{h} b_{h^{\prime}} \in\left(\left(N:_{R} M\right):_{R} s_{g}^{2}\right) \subseteq\left(\left(N:_{R} M\right):_{R} s_{g}\right)$ by Lemma 3.17. Thus $\left(N:_{M} s_{g}\right)$ is a graded 1-absorbing prime submodule of $M$.

(ii) $\Rightarrow$ (i) Assume that $\left(N:_{M} s_{g}\right)$ is a graded 1-absorbing prime submodule of $M$ for some $s_{g} \in S$. Let $a_{h} b_{h^{\prime}} m_{k} \in N$ for some non-unit elements $a_{h}, b_{h^{\prime}} \in h(R)$ and $m_{k} \in h(M)$. As $a_{h} b_{h^{\prime}} m_{k} \in\left(N:_{M} s_{g}\right)$, we get $a_{h} b_{h^{\prime}} \in\left(\left(N:_{M} s_{g}\right):_{R} M\right)$ or $m_{k} \in\left(N:_{M} s_{g}\right)$. Thus $s_{g} a_{h} b_{h^{\prime}} \in\left(N:_{R} M\right)$ or $s_{g} m_{k} \in N$, as needed.

Theorem 3.19. Let $N$ be a graded submodule of $M$ provided $\left(N:_{R} M\right) \subseteq g r-$ $\operatorname{Jac}(R)$, where $g r-\operatorname{Jac}(R)$, the graded Jacobson radical of $R$, is the intersection of graded maximal ideals of $R$. Then the following are equivalent:

(i) $N$ is a graded 1-absorbing prime submodule of $M$.

(ii) $\left(N:_{R} M\right)$ is a graded 1-absorbing prime ideal of $R$ and $N$ is a graded $(h(R)-\mathfrak{m})$-1-absorbing prime submodule of $M$ for each graded maximal ideal $\mathfrak{m}$ of $R$. 
Proof. (i) $\Rightarrow$ (ii) Let $N$ be a graded 1-absorbing prime submodule of $M$. Then $\left(N:_{R} M\right)$ is a graded 1-absorbing prime ideal of $R$, because if $a_{g} b_{h} c_{k} \in\left(N:_{R} M\right)$ and $c_{k} \notin\left(N:_{R} M\right)$ for some $a_{g}, b_{h}, c_{k} \in h(R)$. Thus there exists $m \in M$ such that $c_{k} m \notin N$. Hence $c_{k} m_{g^{\prime}} \notin N$ for some $g^{\prime} \in G\left(m=\sum_{g \in G} m_{g}\right.$ where $\left.m_{g} \in M_{g}\right)$. Therefore, $a_{g} b_{h} \in\left(N:_{R} M\right)$ since $N$ is a graded 1-absorbing prime submodule, as needed. Since $\left(N:_{R} M\right) \subseteq g r-\operatorname{Jac}(R),\left(N:_{R} M\right) \subseteq \mathfrak{m}$ for each graded maximal ideal $\mathfrak{m}$ of $R$, and so $\left(N:_{R} M\right) \cap(h(R)-\mathfrak{m})=\emptyset$. The rest follows from Example 3.3 .

(ii) $\Rightarrow$ (i) Suppose that $\left(N:_{R} M\right)$ is a graded 1-absorbing prime ideal of $R$ and $N$ is a graded $(h(R)-\mathfrak{m})$-1-absorbing prime submodule of $M$ for each graded maximal ideal $\mathfrak{m}$ of $R$. Let $a_{h} b_{h^{\prime}} m_{k} \in N$ with $a_{h} b_{h^{\prime}} \notin\left(N:_{R} M\right)$ for some nonunit elements $a_{h}, b_{h^{\prime}} \in R$ and $m_{k} \in M$. Let $\mathfrak{m}$ be a graded maximal ideal of $R$. Since $N$ is a graded $(h(R)-\mathfrak{m})$-1-absorbing prime submodule of $M$, there exists an $s_{\mathfrak{m}} \in h(R)-\mathfrak{m}$ such that $s_{\mathfrak{m}} a_{h} b_{h^{\prime}} \in\left(N:_{R} M\right)$ or $s_{\mathfrak{m}} m_{k} \in N$. Hence $s_{\mathfrak{m}} a_{h} b_{h^{\prime}} \in\left(N:_{R} M\right)$, and since $\left(N:_{R} M\right)$ is a graded 1-absorbing prime ideal, we have $s_{\mathfrak{m}} \in\left(N:_{R} M\right)$ or $a_{h} b_{h^{\prime}} \in\left(N:_{R} M\right)$, a contradiction. Therefore $s_{\mathfrak{m}} a_{h} b_{h^{\prime}} \notin$ $\left(N:_{R} M\right)$, and hence $s_{\mathfrak{m}} m \in N$. Consider the set $Q=\left\{s_{\mathfrak{m}} \mid s_{\mathfrak{m}} \notin \mathfrak{m}\right.$ and $s_{\mathfrak{m}} m_{k} \in$ $N$ for some graded maximal ideal $\mathfrak{m}$ of $R$. Suppose that $\langle Q\rangle \neq R$. Take any graded maximal ideal $\mathfrak{m}^{\prime}$ containing $Q$ (see [3]). Then the definition of $Q$ requites that there exists $s_{\mathfrak{m}^{\prime}} \in Q$ and $s_{\mathfrak{m}^{\prime}} \notin \mathfrak{m}^{\prime}$, which is a contradiction. Thus $\langle Q\rangle=R$, and so $1=r_{1} s_{\mathfrak{m}_{1}}+r_{2} s_{\mathfrak{m}_{2}}+\cdots+r_{n} s_{\mathfrak{m}_{n}}$ for some $r_{i} \in R$ and $s_{\mathfrak{m}_{i}} \notin \mathfrak{m}_{i}$ with $s_{\mathfrak{m}_{i}} m_{k} \in N$, where $\mathfrak{m}_{i}$ is a graded maximal ideal of $R$ for each $i=1,2, \ldots, n$. Therefore, $m_{k}=r_{1} s_{\mathfrak{m}_{1}} m_{k}+r_{2} s_{\mathfrak{m}_{2}} m_{k}+\cdots+r_{n} s_{\mathfrak{m}_{n}} m_{k} \in N$. Hence $N$ is a graded 1-absorbing prime submodule of $M$.

By the previous theorem we have the following result:

Corollary 3.20. Let $M$ be a graded module over a graded quasilocal ring $(R, \mathfrak{m})$. Then the following are equivalent:

(i) $N$ is a graded 1-absorbing prime submodule of $M$.

(ii) $\left(N:_{R} M\right)$ is a graded 1-absorbing prime ideal of $R$ and $N$ is a graded $(h(R)-\mathfrak{m})$-1-absorbing prime submodule of $M$.

\section{Graded $S$-1-absorbing prime submodules in graded multiplication modules}

In this section, we study graded $S$-1-absorbing prime submodules in graded multiplication $R$-modules. 
Theorem 4.1. Let $S \subseteq h(R)$ be a multiplicatively closed subset of $R$ and $M$ be a faithful graded multiplication $R$-module. Let $I$ be a graded $S$-1-absorbing prime ideal of $R$. Then there exists an $s_{g} \in S$ such that whenever $a_{h} b_{h^{\prime}} m_{k} \in I M$, then either $s_{g} a_{h} b_{h^{\prime}} \in I$ or $s_{g} m_{k} \in I M$ for all non-units $a_{h}, b_{h^{\prime}} \in h(R)$ and all $m_{k} \in h(M)$.

Proof. As $I$ is a graded $S$-1-absorbing prime ideal of $R$, there exists an $s_{g} \in S$ such that whenever $a_{h} b_{h^{\prime}} c_{h^{\prime \prime}} \in I$, then either $s_{g} a_{h} b_{h^{\prime}} \in I$ or $s_{g} c_{h^{\prime \prime}} \in I$ for all non-unit elements $a_{h}, b_{h^{\prime}}, c_{h^{\prime \prime}} \in h(R)$. Choose non-units $a_{h}, b_{h^{\prime}} \in h(R)$ and $m_{k} \in h(M)$ such that $a_{h} b_{h^{\prime}} m_{k} \in I M$ and $s_{g} a_{h} b_{h^{\prime}} \notin I$. Let us define $J=\left\{r \in R: r s_{g} m_{k} \in I M\right\}$. It is easy to see that $J$ is a graded ideal of $R$. If $J=R$, it is done. If $J \neq R$, there is a graded maximal ideal $Q$ of $R$ such that $J \subseteq Q$. We claim that $m_{k} \notin T_{Q}(M)$. Indeed, if $m_{k} \in T_{Q}(M)$, there is an element $q_{g^{\prime}} \in h(R) \backslash Q$ such that $q_{g^{\prime}} m_{k}=0$. This means that $q_{g^{\prime}} \in J \subseteq Q$, a contradiction. Thus $T_{Q}(M) \neq M$. Since $M$ is a graded multiplication module, $M$ is a graded $Q$-cyclic module by Theorem 5.9 in [5]. Hence there is $q_{g^{\prime}}^{\prime} \in h(R) \backslash Q$ and $m_{k^{\prime}}^{\prime} \in h(M)$ such that $q_{g^{\prime}}^{\prime} M \subseteq$ $R m_{k^{\prime}}^{\prime}$. Then $q_{g^{\prime}}^{\prime} s_{g} m_{k} \in R m_{k^{\prime}}^{\prime}$, there is $r_{t} \in h(R)$ such that $q_{g^{\prime}}^{\prime} s_{g} m_{k}=r_{t} m_{k^{\prime}}^{\prime}$ $\left(t=g^{\prime} g\right)$. Then $q_{g^{\prime}}^{\prime} s_{g} a_{h} b_{h^{\prime}} m_{k}=r_{t} a_{h} b_{h^{\prime}} m_{k^{\prime}}^{\prime} \in I M$ and $q_{g^{\prime}}^{\prime} s_{g} a_{h} b_{h^{\prime}} m_{k} \in R m_{k^{\prime}}^{\prime}$. Thus there is $a^{\prime} \in I$ such that $q_{g^{\prime}}^{\prime} s_{g} a_{h} b_{h^{\prime}} m_{k}=a^{\prime} m_{k^{\prime}}^{\prime}$. Since $r_{t} a_{h} b_{h^{\prime}} m_{k^{\prime}}^{\prime}=a^{\prime} m_{k^{\prime}}^{\prime}$, we obtain $r_{t} a_{h} b_{h^{\prime}}-a^{\prime} \in \operatorname{ann}\left(m_{k^{\prime}}^{\prime}\right)$. On the other hand, $q_{g^{\prime}}^{\prime} M \subseteq R m_{k^{\prime}}^{\prime}$ implies that $q_{g^{\prime}}^{\prime}$ ann $\left(m_{k^{\prime}}^{\prime}\right) M \subseteq \operatorname{Rann}\left(m_{k^{\prime}}^{\prime}\right) m_{k^{\prime}}^{\prime}=0$, that is, $q_{g^{\prime}}^{\prime} \operatorname{ann}\left(m_{k^{\prime}}^{\prime}\right) \subseteq \operatorname{ann}(M)$. As $M$ is faithful, $q_{g^{\prime}}^{\prime}$ ann $\left(m_{k^{\prime}}^{\prime}\right)=0$. Then $q_{g^{\prime}}^{\prime}\left(r_{t} a_{h} b_{h^{\prime}}-a^{\prime}\right)=0$. Thus we have $r_{t} a_{h} b_{h^{\prime}} q_{g^{\prime}}^{\prime}=$ $a^{\prime} q_{g^{\prime}}^{\prime} \in I$. Then $r_{t} a_{h} b_{h^{\prime}} q_{g^{\prime}}^{\prime} \in I$. Here we have two situations for $r_{t} \in h(R)$.

Case 1: Let $r_{t}$ be a unit. Then we have $a_{h} b_{h^{\prime}} q_{g^{\prime}}^{\prime} \in I$. If $q_{g^{\prime}}^{\prime}$ is a unit element of $R$, then $a_{h} b_{h^{\prime}} \in I$, and so $s_{g} a_{h} b_{h^{\prime}} \in I$. This contradicts with assumption $s_{g} a_{h} b_{h^{\prime}} \notin I$. Let $q_{g^{\prime}}^{\prime}$ be non-unit. Since $I$ is graded $S$-1-absorbing prime, $s_{g} a_{h} b_{h^{\prime}} \in I$ (again, it is not possible) or $s_{g} q_{g^{\prime}}^{\prime} \in I$. If $s_{g} q_{g^{\prime}}^{\prime} \in I$, then $q_{g^{\prime}}^{\prime} s_{g} m_{k} \in I M$, so $q_{g^{\prime}}^{\prime} \in J \subseteq Q$, a contradiction.

Case 2: Let $r_{t}$ be a non-unit. If $q_{g^{\prime}}^{\prime}$ is a unit element of $R$, then $r_{t} a_{h} b_{h^{\prime}} \in I$. Since $I$ is graded $S$-1-absorbing prime, $s_{g} a_{h} b_{h^{\prime}} \in I$ (again, it is not possible) or $s_{g} r_{t} \in I$. Then $s_{g} r_{t} m_{k^{\prime}}^{\prime} \in I M$. Since $s_{g} r_{t} m_{k^{\prime}}^{\prime}=q_{g^{\prime}}^{\prime} s_{g} m_{g}$, we have $q_{g^{\prime}}^{\prime} s_{g} m_{k} \in I M$. Thus $q_{g^{\prime}}^{\prime} \in J \subseteq Q$, not possible. Now let $q_{g^{\prime}}^{\prime}$ is a non-unit element. Since $I$ is graded $S$-1-absorbing prime, either $s_{g} r_{t} a_{h} b_{h^{\prime}} \in I$ or $s_{g} q_{g^{\prime}}^{\prime} \in I$. Again, since it is graded $S$-1-absorbing prime, we have $s_{g}^{2} r_{t} \in I$ or $s_{g} a_{h} b_{h^{\prime}} \in I$ or $s_{g} q_{g^{\prime}}^{\prime} \in I$. All possibilities give us a contradiction because of the above explanations and $I \cap S=\emptyset$. As a consequence, $J=R$, that is, $s_{g} m_{k} \in I M$.

Corollary 4.2. Let $S \subseteq h(R)$ be a multiplicatively closed subset of $R, M$ be a faithful graded multiplication $R$-module and $I$ be a graded $S$-1-absorbing prime ideal 
of $R$. If $\left(I M:_{R} M\right) \cap S=\emptyset$, then IM is a graded $S$-1-absorbing prime submodule of $M$.

Proposition 4.3. Let $S \subseteq h(R)$ be a multiplicatively closed subset of $R$ and $M$ be a graded multiplication $R$-module. Then $N$ is a graded $S$-1-absorbing prime submodule of $M$ if and only if $\left(N:_{R} M\right)$ is a graded $S$-1-absorbing prime ideal of $R$.

Proof. If $N$ is a graded $S$-1-absorbing prime submodule of $M$, then $\left(N:_{R} M\right)$ is a graded $S$-1-absorbing prime ideal of $R$ by Proposition 3.8. Conversely, assume that $M$ is a graded multiplication module and $\left(N:_{R} M\right)$ is a graded $S$-1-absorbing prime ideal of $R$. Let $I J K \subseteq N$ for some graded ideals $I, J$ of $R$ and a graded submodule $K$ of $M$. Then $I J\left(K:_{R} M\right) \subseteq\left(I J K:_{R} M\right) \subseteq\left(N:_{R} M\right)$. Thus since $\left(N:_{R} M\right)$ is a graded $S$-1-absorbing prime ideal of $R$, by Corollary 3.12, there exists an $s_{g} \in S$ such that $s_{g} I J \in\left(N:_{R} M\right)$ or $s_{g} K \subseteq N$. Therefore, we have $s_{g} I J \in\left(N:_{R} M\right)$ or $s_{g} K=s_{g}\left(K:_{R} M\right) M \subseteq\left(N:_{R} M\right) M=N$. By Theorem 3.11, $N$ is a graded $S$-1-absorbing prime submodule of $M$.

As a consequence of Proposition 4.3 and Theorem 3.11, we have the following explicit result:

Corollary 4.4. Let $S \subseteq h(R)$ be a multiplicatively closed subset of $R, M$ a graded multiplication $R$-module and $N$ a graded submodule of $M$ with $\left(N:_{R} M\right) \cap S=\emptyset$. Then the following statements are equivalent:

(i) $N$ is a graded $S$-1-absorbing prime submodule of $M$.

(ii) There exists an $s_{g} \in S$ such that whenever $K L T \subseteq N$, then either $s_{g} K L \subseteq$ $N$ or $s_{g} T \subseteq N$ for all graded submodules $K, L, T$ of $M$.

The following theorem states a generalization of Theorem 3.2 in [6].

Theorem 4.5. Let $S \subseteq h(R)$ be a multiplicatively closed subset of $R, M$ a graded finitely generated multiplication $R$-module and $N$ a graded submodule of $M$ with $\left(N:_{R} M\right) \cap S=\emptyset$. Then the following are equivalent:

(i) $N$ is a graded $S$-1-absorbing prime submodule of $M$.

(ii) $\left(N:_{R} M\right)$ is a graded $S$-1-absorbing prime ideal of $R$.

(iii) $N=I M$ for some graded $S$-1-absorbing prime ideal $I$ of $R$ with ann $(M) \subseteq$ $I$.

Proof. (i) $\Rightarrow$ (ii) It follows from Proposition 3.8.

(ii) $\Rightarrow$ (iii) It is obvious. 
(iii) $\Rightarrow$ (i) Let $N=I M$ for some graded $S$-1-absorbing prime ideal of $R$ with $\operatorname{ann}(M) \subseteq I$. Assume that $J_{1} J_{2} K \subseteq N$ for some graded ideals $J_{1}, J_{2}$ of $R$ and some graded submodule $K$ of $M$. We have $J_{1} J_{2}\left(K:_{R} M\right) M \subseteq I M$. As $M$ is a graded finitely generated multiplication module, we get $J_{1} J_{2}\left(K:_{R} M\right) \subseteq I+\operatorname{ann}(M)=I$. Since $I$ is a graded $S$-1-absorbing prime ideal of $R$, by Corollary 3.12, there is an $s_{g} \in S$, so that $s_{g} J_{1} J_{2} \subseteq I \subseteq\left(N:_{R} M\right)$ or $s_{g}\left(K:_{R} M\right) \subseteq I \subseteq\left(N:_{R} M\right)$. Therefore, $s_{g} J_{1} J_{2} \subseteq\left(N:_{R} M\right)$ or $s_{g} K \subseteq N$.

Proposition 4.6. Let $S \subseteq h(R)$ be a multiplicatively closed subset of $R$ and $I, J$ be two graded ideals of $R$ such that $I \subseteq J$. If $J$ is a graded $S$-1-absorbing prime ideal of $R$, then $J / I$ is a graded $S$-1-absorbing prime ideal of $R / I$.

Proof. Let $J$ be a graded $S$-1-absorbing prime ideal of $R$. Let $\left(a_{h}+I\right)\left(b_{h^{\prime}}+\right.$ $I)\left(c_{h^{\prime \prime}}+I\right) \in J / I$ for some non-unit elements $\left(a_{h}+I\right),\left(b_{h^{\prime}}+I\right),\left(c_{h^{\prime \prime}}+I\right) \in h(R / I)$. Thus $a_{h} b_{h^{\prime}} c_{h^{\prime \prime}} \in J$. As $\left\{r_{g}+I \mid r_{g} \in U^{g r}(R)\right\} \subseteq U^{g r}(R / I)$, where $U^{g r}(R)$ is the set of unit homogeneous elements of $R$, we obtain $a_{h}, b_{h^{\prime}}, c_{h^{\prime \prime}}$ are non-unit elements. Since $J$ is graded $S$-1-absorbing prime, there exists an $s_{g} \in S$ such that either $s_{g} a_{h} b_{h^{\prime}} \in J$ or $s_{g} c_{h^{\prime \prime}} \in J$. We conclude $s_{g} a_{h} b_{h^{\prime}}+I \in J / I$ or $s_{g} c_{h^{\prime \prime}}+I \in J / I$, as requests.

Definition 4.7. Let $I$ be a graded ideal of $R$. We say that $R$ has the good unit homogeneous element property for $I$, if we have the following equation:

$$
U^{g r}(R / I)=\left\{r_{g}+I: r_{g} \in U^{g r}(R)\right\} .
$$

Now we have the following proposition:

Proposition 4.8. Let $S \subseteq h(R)$ be a multiplicatively closed subset of $R, I, J$ be two graded ideals of $R$ such that $I \subseteq J$ and $R$ has the good unit homogeneous element property for $I$. Then $J$ is a graded $S$-1-absorbing prime ideal of $R$ if and only if $J / I$ is a graded $S$-1-absorbing prime ideal of $R / I$.

Proposition 4.9. Let $S \subseteq h(R)$ be a multiplicatively closed subset of $R, M$ be a graded $R$-module and $R$ has the good unit homogeneous element property for ann $(M)$. If $N$ is a graded $S$-1-absorbing prime submodule as $R /$ ann $(M)$-module $M$, then $N$ is a graded $S$-1-absorbing prime submodule of graded $R$-module $M$.

Proof. Let $a_{h} b_{h^{\prime}} m_{k} \in N$ for some non-units $a_{h}, b_{h^{\prime}} \in h(R)$ and $m_{k} \in h(M)$. Then $\left(a_{h}+\operatorname{ann}(M)\right)\left(b_{h^{\prime}}+\operatorname{ann}(M)\right) m_{k}=a_{h} b_{h^{\prime}} m_{k} \in N$. Since $R$ has the good unit homogeneous element property for $\operatorname{ann}(M)$, we get $a_{h}+\operatorname{ann}(M)$ and $b_{h^{\prime}}+\operatorname{ann}(M)$ are non-unit homogeneous elements of $R / \operatorname{ann}(M)$. As $N$ is a graded $S$-1-absorbing 
prime submodule of $M$ as $R / \operatorname{ann}(M)$-module, there exists an $s_{g} \in S$ such that either $s_{g} a_{h} b_{h^{\prime}}+\operatorname{ann}(M) \in\left(N:_{R / \operatorname{ann}(M)} M\right)$ or $s_{g} m_{k} \in N$. We obtain $s_{g} a_{h} b_{h^{\prime}} M \subseteq$ $N$, that is, $s_{g} a_{h} b_{h^{\prime}} \in\left(N:_{R} M\right)$ or $s_{g} m_{k} \in N$, as required.

In the following theorem, we state Theorem 4.5 under other conditions.

Theorem 4.10. Let $S \subseteq h(R)$ be a multiplicatively closed subset of $R, M$ be a graded multiplication $R$-module, $R$ have the good unit homogeneous element property for ann $(M)$ and $N$ be a graded submodule of $M$ with $\left(N:_{R} M\right) \cap S=\emptyset$. Then the following are equivalent:

(i) $N$ is a graded $S$-1-absorbing prime submodule of $M$.

(ii) $\left(N:_{R} M\right)$ is a graded $S$-1-absorbing prime ideal of $R$.

(iii) $N=I M$ for some graded $S$-1-absorbing prime ideal $I$ of $R$ with ann $(M) \subseteq$ $I$.

Proof. (i) $\Rightarrow$ (ii) It follows from Proposition 3.8.

(ii) $\Rightarrow$ (iii) Consider $I=\left(N:_{R} M\right)$.

(iii) $\Rightarrow$ (i) Since $M$ is a graded multiplication $R$-module, $M$ is faithful graded multiplication $R / \operatorname{ann}(M)$-module. As $I$ is a graded $S$-1-absorbing prime ideal of $R, I / \operatorname{ann}(M)$ is a graded $S$-1-absorbing prime ideal of $R / \operatorname{ann}(M)$, see Proposition 4.6. Thus $(I / \operatorname{ann}(M)) M$ is a graded $S$-1-absorbing prime submodule of graded $R / \operatorname{ann}(M)$-module $M$, by Corollary 4.2. This means that $(I / \operatorname{ann}(M)) M$ is a graded $S$-1-absorbing prime submodule of graded $R$-module $M$, by Proposition 4.9. As $(I / \operatorname{ann}(M)) M=N$, we get $N$ is a graded $S$-1-absorbing prime submodule of graded $R$-module $M$.

Acknowledgement. The authors would like to thank the referee for the valuable suggestions and comments.

\section{References}

[1] R. Abu-Dawwas, M. Bataineh and M. Al-Muanger, Graded prime submodules over non-commutative rings, Vietnam J. Math., 46(3) (2018), 681-692.

[2] K. Al-Zoubi and R. Abu-Dawwas, On graded 2-absorbing and weakly graded 2-absorbing submodules, Journal of Mathematical Sciences: Advances and Applications, 28 (2014), 45-60.

[3] S. Ebrahimi Atani and F. Farzalipour, Notes on the graded prime submodules, Int. Math. Forum, (1) (2006), 1871-1880.

[4] S. Ebrahimi Atani and F. Farzalipour, On graded secondary modules, Turkish J. Math., 31 (2007), 371-378. 
[5] J. Escoriza and B. Torrecillas, Multiplication objects in commutative Grothendieck categories, Comm. Algebra, 26(6) (1998), 1867-1883.

[6] F. Farzalipour and P. Ghiasvand, On S-1-absorbing prime submodules, J. Algebra Appl., to appear.

[7] F. Farzalipour and P. Ghiasvand, On the union of graded prime submodules, Thai. J. Math., 9(1) (2011), 49-55.

[8] F. Farzalipour and P. Ghiasvand, On graded weak multiplication modules, Tamkang J. Math., 43(2) (2012), 171-177.

[9] F. Farzalipour, P. Ghiasvand and M. Adlifard, On graded weakly semiprime submodules, Thai. J. Math., 12(1) (2014), 167-174.

[10] P. Ghiasvand and F. Farzalipour, Some properties of graded multiplication modules, Far East J. Math. Sci., 34(3) (2009), 341-352.

[11] P. Ghiasvand and F. Farzalipour, On the graded primary radical of graded submodules, Adv. Appl. Math. Sci., 10(1) (2011), 1-7.

[12] K. H. Oral, Ü. Tekir and A. G. Ağargün, On graded prime and primary submodules, Turkish J. Math., 35 (2011), 159-167.

[13] N. Nastasescu and F. van Oystaeyen, Graded Ring Theory, North-Holland Mathematical Library, North-Holland Publishing Co., Amsterdam-New York, 1982.

[14] N. Nastasescu and F. van Oystaeyen, Methods of Graded Rings, Lecture Notes in Mathematics, vol. 1836., Springer-Verlag, Berlin, 2004.

F. Farzalipour (Corresponding Author) and P. Ghiasvand

Department of Mathematics

Payame Noor University

P .O. BOX 19395-3697 Tehran, Iran

e-mails: f_farzalipour@pnu.ac.ir (F. Farzalipour)

p_ghiasvand@pnu.ac.ir (P. Ghiasvand) 